\title{
BMJ Quality A Quality Improvement Project to Reduce the 'No Show' rate in a Paediatric Neurology Clinic
}

\author{
Khalid Mohamed, Amira Mustafa, Sona Tahtamouni, Eshraga Taha, \\ Reham Hassan
}

To cite: Mohamed K, Mustafa A, Tahtamouni S, et al. A Quality Improvement Project to Reduce the 'No Show' rate in a Paediatric Neurology Clinic. BMJ Quality Improvement Reports 2016;5:u209266.w3789 doi:10.1136/bmjquality. u209266.w3789

Received 25 February 2016 Revised 1 June 2016

\section{ABSTRACT}

This quality improvement project aimed to reduce the 'no show' rate in a paediatric neurology clinic in Qatar.

No show, in outpatient clinics, is defined as patients who fail to attend their scheduled clinic appointments. It is one of the targets for improving quality of care. It leads to longer waiting times for patients to be seen in outpatient clinics, and the result is patients missing their important appointments. It also results in a waste of the clinic resources, and physician and other healthcare practitioners' time.

This study was undertaken as part of the CCITP (clinical care improvement training programme). A project team was assembled with coaching support. The department chairman and the appointment system personnel were involved. Baseline and ongoing measures were collected and charted.

The baseline no-show rate was identified as $49 \%$. Following three intervention PDSAs, mainly addressing communication and appointment flexibility, the post intervention no-show rate dropped to $18 \%$ and was sustained below the target of $25 \%$ for two years.

Better communication and appointment flexibility can significantly reduce the no-show rate in outpatient clinics.

\section{PROBLEM}

In the Paediatric Neurology Clinic at Hamad General Hospital, Qatar, $49 \%$ of patients referred to pediatric neurology did not show up for their appointments leading to long waiting times for new patients, and a waste of clinic resources. The international bench-mark for primary care clinics is 5-10\%, however for specialist healthcare centres, the no-show rate has been reported to be higher. ${ }^{12}$

\section{BACKGROUND}

No-show in outpatient clinics, also known as DNA (Did Not Attend) is a cause of significant concern for healthcare providers. Patients don't attend appointments for several reasons including; logistical reasons such as transport difficulties or the lack of a suitable carer to bring the child. There are also appointment related reasons including; scheduling, communication, and timing of the appointment.

A recent study by DuMontier et al, ${ }^{3}$ from Wisconsin, showed that effective interventions reduced the no-show rate in both the general population and the chronic/habitual no-show cohort. Changing clinic schedules can be effective if it is adopted to a specific population, but can be cumbersome in a large health care facility.

Izard $^{4}$ reported on a Quality improvement system implemented in Milwaukee that included focused interventions for habitual no-show patients and resulted in a $20 \%$ drop in the no-show rate. Similar work in Psychiatry from Gajwani ${ }^{5}$ in Texas resulted in a significant and maintained reduction of the no-show rate.

Learning from the literature, it is clear that multi faceted interventions aimed at all levels of the process have a high success rate. Johnson et $\mathrm{al}^{6}$ describe how communicating when conducting outpatient appointments is crucial if the no show rate is to be managed and reduced effectively.

\section{BASELINE MEASUREMENT}

No-show was measured as the percentage of patients who did not attend their appointments, compared to all patients given appointments for one paediatric neurology outpatient clinic.

A record of all appointments given, cancellations, and patients who did not attend was kept by the clinic nurse and checked by the physician.

In the first six weeks of the project, 26 patients out of $53(49 \%)$ did not attend or cancelled their clinic appointments. Six more patients $(11 \%)$ cancelled their clinic appointments but only half of them were replaced by other patients as the other half 
did not give adequate notice of cancellation for the appointment office to notify a replacement.

The median waiting time to the third available appointment was 11 weeks. We used this as an indicator of the clinic's efficiency. This measure is affected by the high no show rate, as patients who fail to attend their appointments are rescheduled, which affects the waiting time.

\section{DESIGN}

The project was developed as part of the learning platform of the Clinical Care Improvement Training Programme. The project had coaching support. The team was composed of physicians, a clinic nurse, a specialist nurse, contact centre staff, and the department secretary. The team drew a process map of how patients are given and notified of their appointments. A fishbone was also drawn to analyse the root causes and three interventions were identified as suitable for PDSA which were;

- Improving the way we contact parents, allowing them to ask for an immediate re-book if they know they can not make the appointment.

- A review of all available slots on the morning before the clinic date and replacing cancelled slots by patients from the urgent waiting list.

- The physician reviews the notes for the habitual no-show patients (more than once) and decides whether they need further appointments, otherwise they can be contacted by the specialty nurse.

\section{STRATEGY}

PDSA Cycle1 (September 2013)

Aim: To reduce the no show rate for the Pediatric Neurology outpatient clinic at Hamad General Hospital from $49 \%$ to $25 \%$ by December 31st 2013 (4 months)
Plan: Offer patients an immediate re-book by asking them whether the appointment time is suitable or not.

Prediction: Many patients would re-book or cancel the appointment instead of accepting it and then not attending.

Do: to our surprise around $40 \%$ of all patients requested a re-book or informed us that they don't need the appointment

Study: The results confirmed this as a crucial intervention as the no show rate dropped to $33 \%$ in two weeks.

Act: We implemented the new call structure for all clinics within the facility and defined cancellation slots as our target for the next PDSA.

PDSA Cycle 2 (October 2013)

Aim: To reduce the no show rate for the Pediatric Neurology outpatient clinic at Hamad General Hospital from $49 \%$ to $25 \%$ by December 31st 2013 (4 months)

Plan: Implement a system where all cancelled appointments are refilled in the morning before the clinic form the list of urgent patients, by liaison between the clinician and the patient referral service.

Prediction: Patients from the urgent list are likely to come to appointments as they have been referred recently. This will also improve the overall waiting time which will impact on the no-show rate.

Do: There was a further improvement in the no show rate and the urgent list reduced immediately.

Study: The results confirmed this as a crucial intervention, with this the no show rate dropping to our target of $25 \%$.

Act: We identified the habitual no-show patients as a target for the next PDSA.

PDSA Cycle 3: (October-November 2013)

Aim: To reduce the no show rate for the Pediatric Neurology outpatient clinic at Hamad General Hospital from $49 \%$ to $25 \%$ by December 31st 2013 (4 months)

\section{Cause and Effect Diagram}

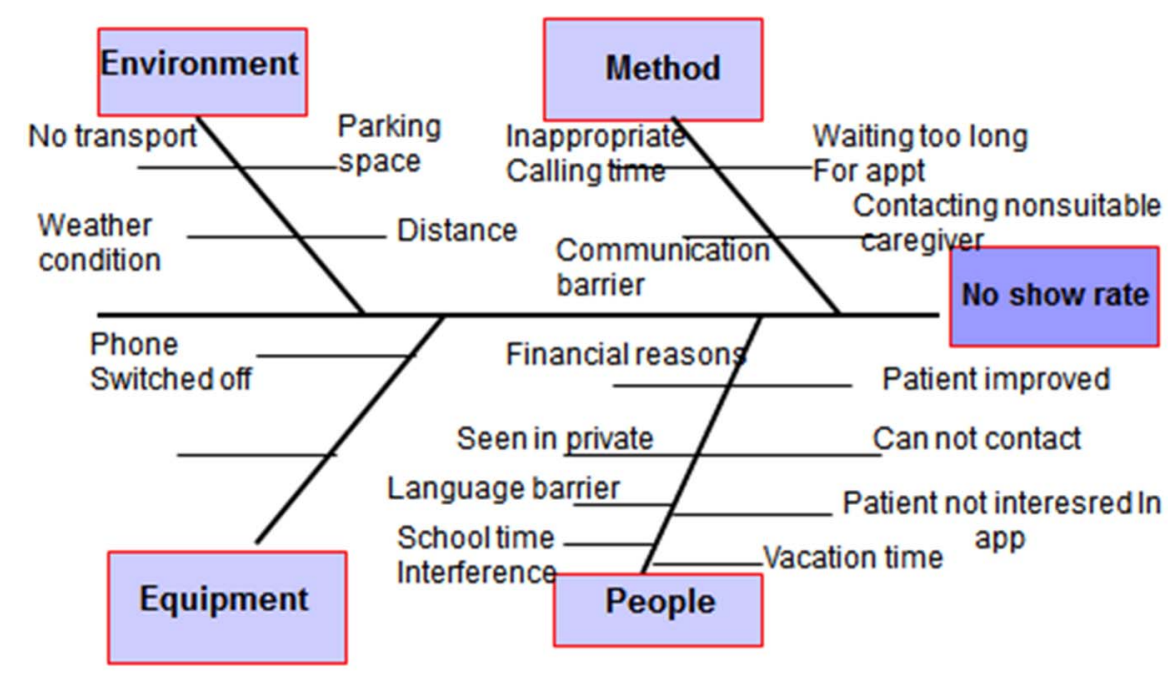


Plan: To discharge patients who failed to attend twice.

Prediction: Around 25\% of our no show patients failed to attend for the third time. Our appointment system used to offer patients three appointments before discharging them from the service. We found that less than $10 \%$ of patients who failed to attend twice, turned up for the third appointment. We predicted that by discharging patients after the second non-attendance we would reduce the no show rate further.

Do: Patients who failed to attend twice were discharged from the clinic and removed from the list. A note was send to the referrer to inform them that their patient did not show twice.

Study: Our no show rate reduced further to $18 \%$.

Act: This was implemented as a standard.

\section{RESULTS}

After the 3rd PDSA cycle, the no-show rate dropped to $18 \%$ in the post intervention period. This improvement was sustained for 24 months following the completion of the project by continued liaison between the responsible physician and the patient referral and call centre. There was an effort to spread this across all outpatient clinics in the department. The effect of the changed call structure also resulted in an improvement in the attendance to other clinics within the department.

Median waiting time to the third available appointment reduced to eight weeks (30\% improvement).

\section{LESSONS AND LIMITATIONS}

It is possible to reduce the no-show rate by implementing changes to the appointment system and patient communication strategies.

When we reviewed the process of booking and communicating appointments to the parents, many of the team's misconceptions were immediately rectified. This was due to the team walking in the patient/parents shoes to fully understand the process.

The success of the first intervention identified another area for improvement as the number of cancellations created space for urgent patients. This in turn further improved attendance and reduced the overall waiting time for clinic appointments.

A limitation of this improvement project is that there were some cultural issues which made it difficult to achieve the international benchmark of 5-10\%; patients sometimes had issues with taking time off work and providing carers to bring their children to their appointments.

\section{CONCLUSION}

We conclude that improved strategies for communications with parents, a flexible appointment system and better communication between the clinical and admin teams resulted in a significant reduction in the no show rate in this busy paediatric neurology clinic. There were also associated benefits of improving access for urgent patients and a reduction in the overall waiting time.

\section{Baseline Process Map

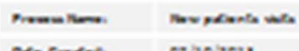

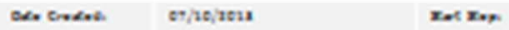

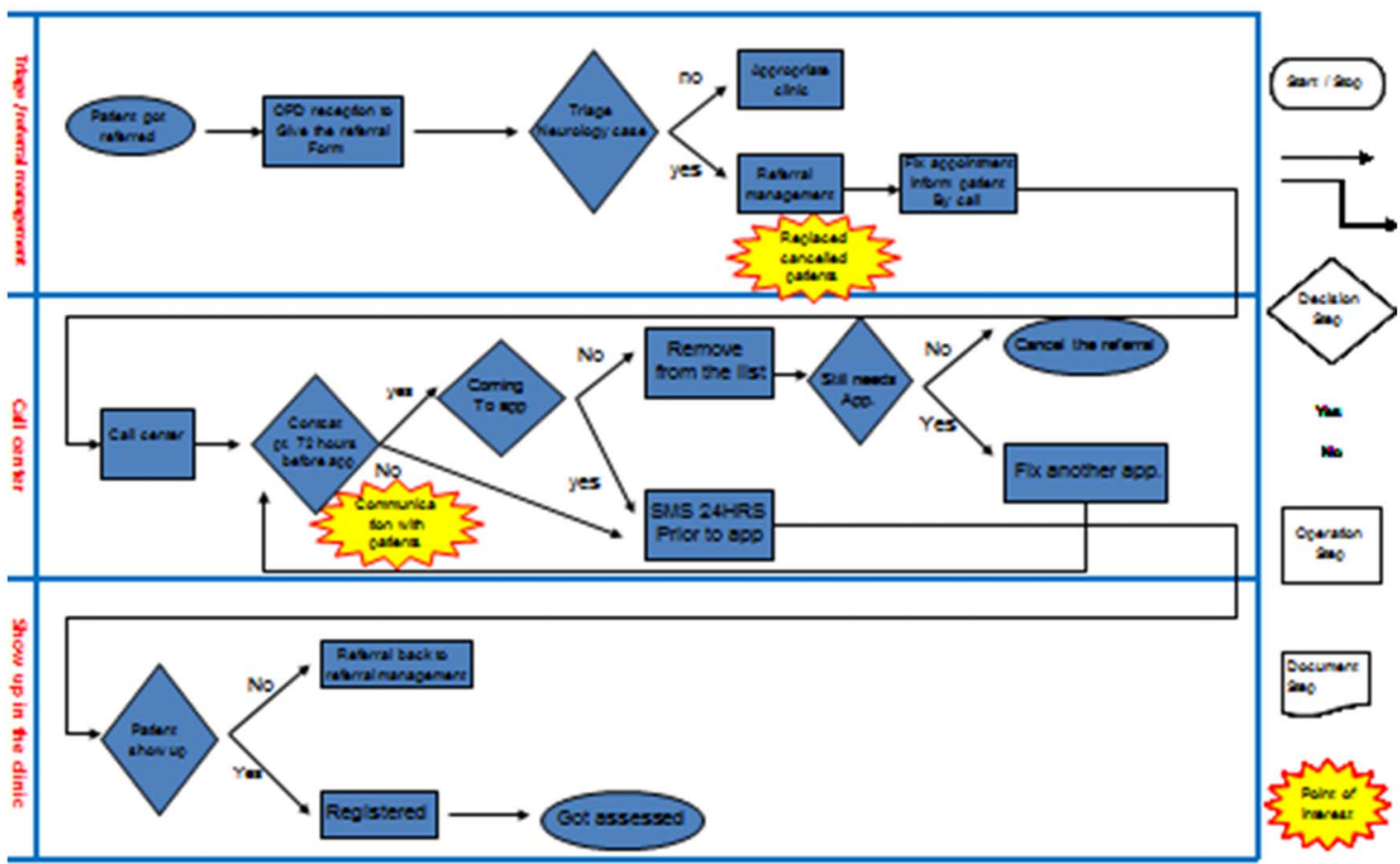




\section{Percentage of no show to Pediatric Neurology clinics}

HMC-Qatar

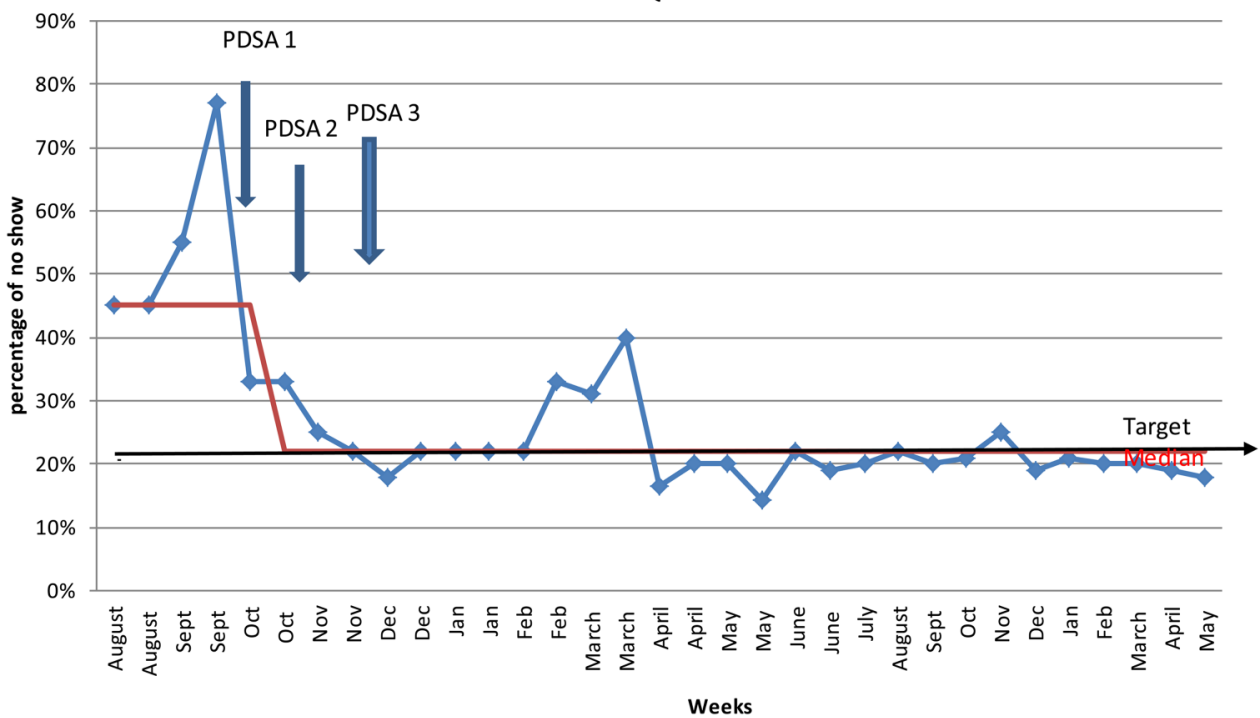

Sustaining the gains required vigilance and regular audit of the outcome measure, which is currently one of the key performance indicators for paediatric neurology. A feedback loop was created for the clinical team to inform the booking services when the no show rate goes up to rectify the process.

Acknowledgements Mohammad Janahi Pediatric Chairman, Zeenia Kersi, Amel Chehab project team members.

Declaration of interests Nothing to declare

Ethical approval This work was deemed an improvement study and not a research study on human subjects as patients were not affected directly and their treatment was not altered nor were they given an experimental or novel therapy, the local research policy meant that ethical approval was not required. This was confirmed by the IRB.

Open Access This is an open-access article distributed under the terms of the Creative Commons Attribution Non-commercial License, which permits use, distribution, and reproduction in any medium, provided the original work is properly cited, the use is non commercial and is otherwise in compliance with the license. See:

- http://creativecommons.org/licenses/by-nc/2.0/

- http://creativecommons.org/licenses/by-nc/2.0/legalcode

\section{REFERENCES}

1. Molfentera T. Reducing Appointment No-Shows: Going from Theory to Practice. Substance Use \& Misuse 48:2013

2. Gupta D, Denton B. Appointment scheduling in health care: Challenges and opportunities IIE. Transactions 40:2008.

3. DuMontier C, Rindfleisch K, Pruszynski J, Frey III J. A Multi-Method Intervention to Reduce No-Shows in an Urban Residency Clinic. Fam Med 2013;45:634-41

4. Izard DT. Managing the habitual no-show patient. American Academy of Family Physicians online publication (2005).

5. Gajwani P. Can what we learned about reducing no-shows in our clinic work for you? Current Psychiatry 2014 September; 13:13-15, 22-14.

6. Johnson B, Mold J, Pontious M. Reduction and Management of No-Shows by Family Medicine Residency Practice Exemplars. Ann Fam Med. 2007 Nov; 5: 534-539. 